\title{
THE EQUATION OF LOAD DEFORMATION DIAGRAMS
}

\section{Duishenaliev, T.; Duishenaliev, C. \& MeKenbaev, B.}

Abstract: The results of experiments under axial compression and tension were described by defined ratios. These ratios are derived from the solution of the boundary problem of equilibrium of the cylinder, the boundary conditions on the bases of which are simplified by reducing the components a general principle Saint-Venant. These equations do not generalize Hooke's law, this is view of the equation in which is invisibly are present these principles. Bearing this in mind, these equations should be called relationships Hooke - Saint-Venant. The deviation of the experimental data from this relationship should be discussed not as a deviation from the Hooke's generalized law as well its deviation from simplified version of equation. Simplified principle of Saint Venant has a theoretical proof. A general principle of such evidence has not. Numerous attempts to prove its principle are known to have not succeeded. In this paper carried out the integration of equation of axial movement, at which the used the generalized Hooke's law and the boundary conditions on the grounds and the lateral surface of a cylindrical sample. This equation at the same values of the constants a good description of the experimental data at all levels of deformation.

Key words: general principle Saint-Venant, Hooke's law, experiments
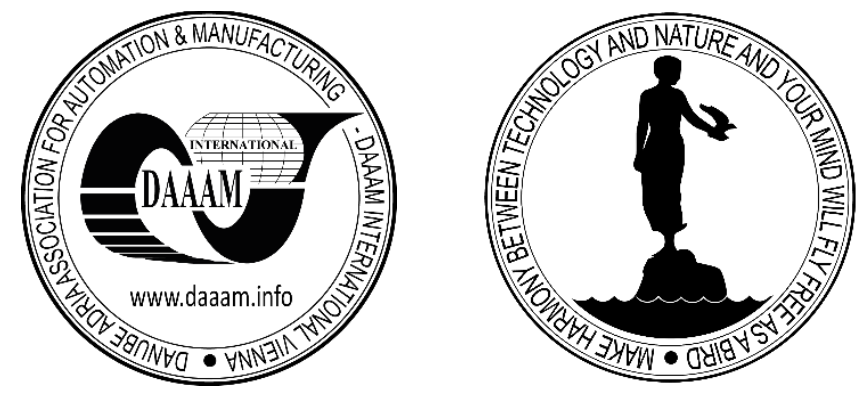

Authors' data: Univ.Prof. Dipl.-Ing. Dr.techn. Duishenaliev, T[uratbek]; Dipl.-Ing. Duishenaliev, $\mathrm{Ch}[$ ingiz]; Dipl.-Ing. Mekenbaev, B[aktybek], *Kyrgyz State Technical University, Mira avenue 66, 720044, Bishkek, Kyrgyzstan, duishenaliev@mail.ru

This Publication has to be referred as: Duishenaliev, T[uratbek]; Duishenaliev, C[hingiz] \& Mekenbaev, B[aktybek] (2017). The Equation of Load Deformation Diagrams, Chapter 15 in DAAAM International Scientific Book 2017, pp.191-208, B. Katalinic (Ed.), Published by DAAAM International, ISBN 978-3-902734-12-9, ISSN 1726-9687, Vienna, Austria

DOI: $10.2507 /$ daaam.scibook.2017.15 


\section{Introduction}

The theoretical mechanics is learnt the movement of bodies in the space based on of a uniform equation - law of Newton. The theoretical mechanics is an exact science. Let us admit, for some time, the law of Newton is unknown and this science uses other kinds of a ratio between force and acceleration. The real chaos would be established in it then! It would turn to an opus consisting of various sections, where the different ratio between force and acceleration are put in their base. Infinite comparisons of various descriptions would be carried out, which conclusions will be right and which will be wrong would be identified. In other words, the theoretical mechanics would become a fertile field for researches and promotions of any sort of hypotheses. Here we do not exaggerate at all. Such state of affairs is natural to any disciplines that are not having the organic law. The rigid body mechanics is one of them. It is considered that there is no the uniform conventional law in the mechanics, connecting among themselves tensor components of a stress and deformation. Such law is searched from sources of development of this science up to now. Search of this law always was an actual problem that was a subject of researches of the greatest mathematicians, mechanics and physicists of several generations (Lagrange, Cauchy, Poisson, Ljav, Maxwell, Einstein and Born).

Among the ratio connecting among themselves tensor components of a stress and deformation, the special place occupies generalized Hooke's law. This law has a strict physical substantiation. It is deduced from modern understanding of molecular structure of the bodies (M. Born, Huan Kun, 1958). It is possible to come to this law from thermodynamics as well. Equations of Duhamel - Neumann and the equations received from Gibbs potential, and functions of conditions of internal energy in isothermal processes, turn to a kind of ratio of this law.

In the given work the solvency of this law is investigated. It is shown, that it has that solvency which is inherent to the fundamental laws of the nature.

\section{A. Experimental data were measured on the basis of the generalized Hooke's law}

Let's consider symmetrical axis compression, a stretching and three-axis compression of a cylindrical sample. Boundary conditions are submitted on figure 1 .

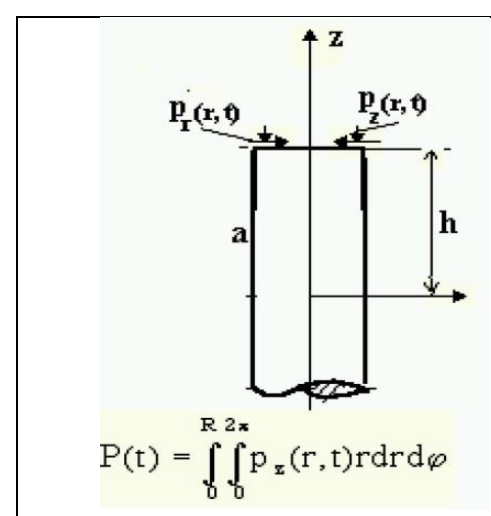

a)

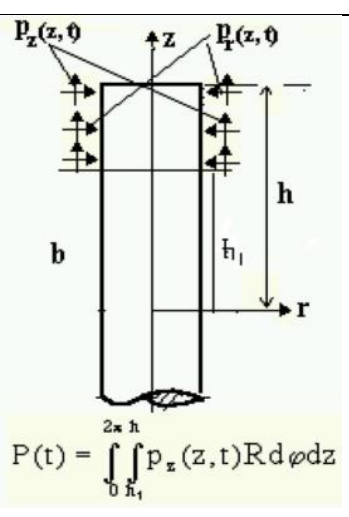

b)

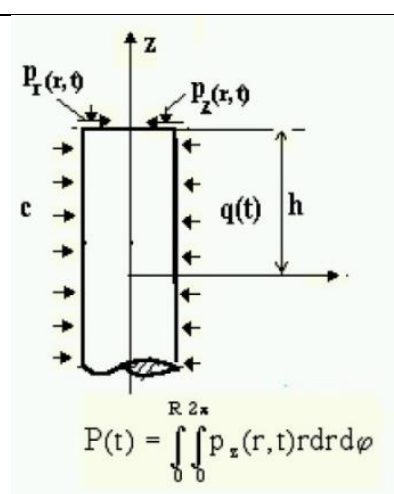

c)

Fig. 1. Boundary conditions in axial compression, a stretching and three-axis compression of a cylindrical sample 


$$
\int_{0}^{R} \int_{0}^{2 \pi} \int_{z}^{h}\left(\frac{\partial \sigma_{z r}}{\partial r}+\frac{\partial \sigma_{z}}{\partial z}+\frac{\sigma_{r}}{r}\right) r d r d \varphi d \xi=\int_{0}^{R} \int_{0}^{2 \pi} \int_{z}^{h} \rho \frac{\partial^{2} w}{\partial t^{2}} r d r d \varphi d \xi
$$

Let's write (1) as

$$
\int_{0}^{2 \pi} \int_{z}^{h} d \varphi d \xi \int_{0}^{R} \frac{\partial}{\partial r}\left(r \sigma_{z r}\right) d r+\int_{0}^{R} \int_{0}^{2 \pi} r d r d \varphi \int_{z}^{h} \frac{\partial \sigma_{z}}{\partial \xi} d \xi=\int_{0}^{R} \int_{0}^{2 \pi} \int_{z}^{h} \rho \frac{\partial^{2} w}{\partial t^{2}} r d r d \varphi d \xi
$$

This expression allows leaving to the basis $(\mathrm{z}=\mathrm{h})$ and a lateral surface of the cylinder $(\mathrm{r}=\mathrm{R})$. Using boundary conditions and representations for the axial loading, specified on figure 1, in all three cases we come to the equation:

$$
P(t)=2 \pi \int_{0}^{R} \sigma_{z} r d r+\int_{0}^{R} \int_{0}^{2 \pi} \int_{z}^{h} \rho \frac{\partial^{2} w}{\partial t^{2}} r d r d \varphi d \xi
$$

Let's estimate inertial addendum. Let $\rho=10 \mathrm{t} / \mathrm{m}^{3}, \mathrm{R}=\mathrm{h}=40 \mathrm{~mm},\left|\frac{\partial^{2} w}{\partial t^{2}}\right|_{\max }=1 \mathrm{~m} / \mathrm{sec}^{2}$. Size of last integral at such huge acceleration for usual tests

$$
\int_{0}^{R} \int_{0}^{2 \pi} \int_{z}^{h} \rho \frac{\partial^{2} w}{\partial t^{2}} r d r d \varphi d \xi \leq \rho\left|\frac{\partial^{2} w}{\partial t^{2}}\right|_{\max } \frac{R^{2}}{2} 2 \pi(h-z) \leq \rho\left|\frac{\partial^{2} w}{\partial t^{2}}\right|_{\max } \pi R^{2} h=2 H
$$

It is rather small size in comparison with size of the axial loading estimated in $104 \mathrm{H}$. From here it is visible, that inertial addendum may be realized by increasing acceleration in ten thousand times, as it is marked in the literature. By virtue of it, representation of exact expression (2) as

$$
P(t)=2 \pi \int_{0}^{R} \sigma_{z} r d r
$$

won't lead to a little appreciable mistake and at very fast loadings.

According to the generalized Hooke's law

$$
\sigma_{z}=\lambda\left(\frac{\partial u}{\partial r}+\frac{u}{r}\right)+(\lambda+2 \mu) \frac{\partial w}{\partial z}
$$

where $\lambda, \boldsymbol{\mu}$ - Lame constants.

Let's substitute it in (3) and carry out some integration 


$$
P(t)=\pi R^{2}\left(\frac{\lambda(\lambda+2 \mu)}{R^{2}} \int_{0}^{R} \frac{\partial w}{\partial z} r d r+2 \lambda \frac{u(R, z, t)}{R}\right)
$$

Because of symmetry of loading, function $\mathbf{w}(\mathbf{r}, \mathbf{z}, \mathbf{t})$ at $\mathbf{z}=\mathbf{0}$ does not depend from $\mathbf{r}$ $\mathbf{w}(\mathbf{r}, \mathbf{z}, \mathbf{t})=\mathbf{0}$.

On reasons of continuity and at values $\mathbf{z}$, close to zero, dependence of this function from $\mathbf{r}$ should be very weak, that allows making the approach

$$
\frac{\partial w(r, 0, t)}{\partial z}=\frac{\partial w(R, 0, t)}{\partial z}=\varepsilon_{z}(R, 0, t)
$$

Believing in (24) $\mathbf{z}=\mathbf{0}$ and using the previous representation, finally we have

$$
\varepsilon_{z}(R, 0, t)=\frac{1}{\lambda+2 \mu}\left(\frac{P(t)}{\pi R^{2}}-2 \lambda \varepsilon_{\varphi}(R, 0, t)\right)
$$

It's nice that for all three kinds of tests of bodies the same equation was calculated. It represents of using when the experimental data were measured on the basis of the generalized Hooke's law.

This is an exact name of the equation (5), which is received from integral (3) where sub integral expression is expressed by this law. We shall name it Jakypbek's criterion. This criterion gives new interpretation to the diagram loading - deformation of which is shown on figure 2 .

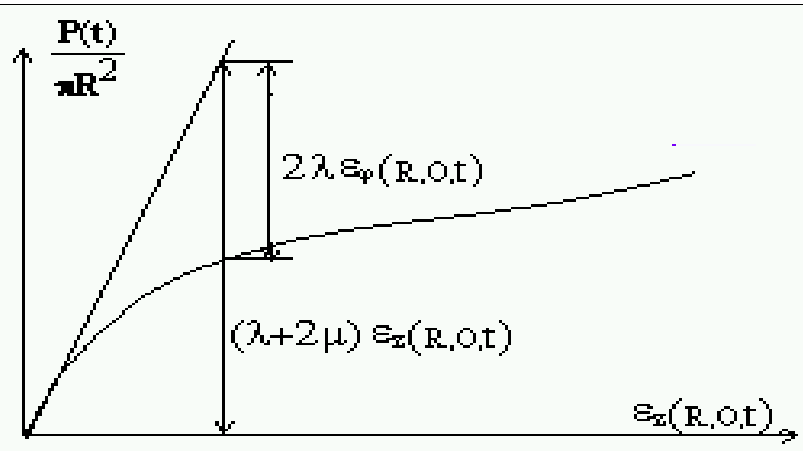

Fig. 2. The new interpretation of diagrams.

For comparison we shall give a conclusion of usual criterion. Let the average part of the cylinder in height $\mathbf{2} \mathbf{h}$ and radius $\mathbf{R}$ is in balance. The decision of a static boundaryvalue problem with boundary conditions: on the top and bottom bases

$$
\sigma_{z r}(r, \pm h)=0, \quad \sigma_{z}(r, h)= \pm R / \pi R^{2}
$$

and on a lateral surface

$\sigma_{r z}(R, z)=0, \sigma_{z}(R, z)=0$, 
functions are

$$
\sigma_{r}(r, z)=\sigma_{\varphi}(r, z)=\sigma_{r z}(r, z)=0, \quad \sigma_{z}(r, z)=P / \pi R^{2}
$$

From expressions (7) ratio follow

$$
\begin{aligned}
& \varepsilon_{r}(r, z)=\varepsilon_{\varphi}(r, z)=-v P /\left(E \pi R^{2}\right), \varepsilon_{r z}(r, z)=0, \\
& \varepsilon_{z}(r, z)=P /\left(E \pi R^{2}\right) .
\end{aligned}
$$

where E, $\boldsymbol{v}$ - Elastic modulus, Poisson's ratio. Results of discussion of a solvency of generalized Hooke's law are known from the point of view of usual criterion (8). What conclusions will be obtained after the discussion of a solvency of generalized Hooke's law from the point of view of new criterion?

\section{Definition the constant of material}

The equation (5) represents only those sizes, which are measured in experiences. In our work, the new methods of constants definitions, which have the best results, are elaborated (in comparison with the method of the least squares). Let's write the equation (5) as

$$
\lambda+2 \mu=\frac{\frac{P(t)}{\pi R^{2}}}{\varepsilon_{z}(R, 0, t)+\frac{2 \lambda}{\lambda+2 \mu} \varepsilon_{\varphi}(R, 0, t)}
$$

We admit, the given material knows the ratio $2 \lambda /(\lambda+2 \mu)$. In this case the size of fraction in the right part (9) should remain a constant at any stage of deformation. In other words, this fraction should have the same size in an initial part both in the field of current and in a zone of strengthening of the experiment diagram. This circumstance was the initial point for program drawing.

The constants contained in the equation (5), may be defined by the least squares method. We admit, that at the moment of time $t_{0}, t_{1}, \ldots, t_{n}$ sizes of loading, ring and axial deformations are measured. We shall write them as matrixes:

$$
p:=\frac{1}{\pi \cdot R^{2}} \cdot\left[P\left(t_{1}\right) P\left(t_{2}\right) \ldots P\left(t_{n}\right)\right] \varepsilon:=\left[\begin{array}{cccc}
\varepsilon_{\phi}\left(R, 0, t_{1}\right) & \varepsilon_{\phi}\left(R, 0, t_{2}\right) & \ldots & \varepsilon_{\phi}\left(R, 0, t_{n}\right) \\
\varepsilon_{z}\left(R, 1, t_{1}\right) & \varepsilon_{z}\left(R, 0, t_{2}\right) & \ldots & \varepsilon_{z}\left(R, 0, t_{n}\right)
\end{array}\right]
$$

Systems Mathcad, MATLAB define constants by one operator 


$$
c:=\left(\varepsilon \cdot \varepsilon^{\mathrm{T}}\right)^{-1} \cdot \varepsilon \cdot p^{\mathrm{T}}
$$

From here $\lambda=c_{0} / 2, \mu=\left(c_{1}-\lambda\right) / 2$.

\section{Definition of radial deformation and stresss on a cross-section counter of the cylinder under its axial stretching and compression}

Radial deformation is not measured at an axial stretching and compression of cylindrical samples because it cannot be carried out. However, the size of this deformation on a lateral surface of the cylinder can be defined from a condition of absence of external efforts on this surface

$$
\sigma_{r}(R, 0, t)=(\lambda+2 \mu) \varepsilon_{r}(R, 0, t)+\lambda\left(\varepsilon_{\varphi}(R, 0, t)+\varepsilon_{z}(R, 0, t)\right)=0
$$

From this boundary condition follows

$$
\varepsilon_{r}(R, 0, t)=-\frac{\lambda}{\lambda+2 \mu}\left(\varepsilon_{\varphi}(R, 0, t)+\varepsilon_{z}(R, 0, t)\right)
$$

Sizes of ring and axial deformation may be measured at all stages of deformation, and radial deformations can be established, using equality (11). Knowing sizes of three components of deformation, it is easy to define sizes of an axial and ring stress. In the above-stated program alongside with the definition of material constants, calculation of radial deformation sizes is made $\varepsilon_{r}(R, 0, t)$, axial $\sigma_{z}(R, 0, t)$ and ring $\sigma_{\varphi}(R, 0, t)$ stress

$$
\begin{aligned}
& \sigma_{\varphi}(R, 0, t)=\lambda \varepsilon_{r}(R, 0, t)+(\lambda+2 \mu) \varepsilon_{\varphi}(R, 0, t)+\lambda \varepsilon_{z}(R, 0, t) \\
& \sigma_{z}(R, 0, t)=\lambda \varepsilon_{r}(R, 0, t)+\lambda \varepsilon_{\varphi}(R, 0, t)+(\lambda+2 \mu) \varepsilon_{z}(R, 0, t)
\end{aligned}
$$

\section{Studying the experimental data on the basis of new criterion}

Axial stretching and compression: After definition of constants by a method of the least squares or by suggested method, it is possible to calculate sizes of radial deformation (11), ring and axial stress (12). Below, in table 1, the experimental data received at a stretching of steel 30 [2], the designed sizes of deformations and stress are given. Dimension of stress $-\mathrm{kgs} / \mathrm{mm} 2$. Sizes of constants in $(\mathrm{kgs} / \mathrm{mm} 2) \lambda=43001, \mu=1413$, $\mathrm{E}=4193$. Poisson's ratio $v=0.4841$.

We shall note, size $v$ here it is determined from sizes $\lambda$, $\mu$, instead of as a ratio $v=-\varepsilon_{\varphi} / \varepsilon_{\mathbf{z}}$ which is consequence of boundary conditions (6). Second of these conditions nevertheless is doubtful, for it is based on general Saint-Venant principle. Attempts of its strict theoretical substantiations, as is known, are not successful yet. In the column 
5 deviations of experimental and designed by the equation (5) are given. Sizes of axial deformation are specified here too.

\begin{tabular}{|c|c|c|c|c|c|c|c|}
\hline$\varepsilon_{\mathrm{r}}(\mathrm{R}, 0, \mathrm{t}) 10^{3}$ & $\varepsilon_{\varphi}(\mathrm{R}, 0, \mathrm{t}) 10^{3}$ & $\varepsilon_{\mathrm{z}}(\mathrm{R}, 0, \mathrm{t}) 10^{3}$ & $\varepsilon_{\mathrm{z}}(\mathrm{R}, 0, \mathrm{t}) 10^{2}$ & $\%$ & $\mathrm{P} / \pi \mathrm{R}^{2}$ & $\sigma_{\mathrm{r}}(\mathrm{R}, 0, \mathrm{t})$ & $\sigma_{\varphi}(\mathrm{R}, 0, \mathrm{t})$ \\
\hline $\begin{array}{c}\text { data from } \\
\text { calculation }\end{array}$ & $\begin{array}{c}\text { data from } \\
\text { experiment }\end{array}$ & $\begin{array}{c}\text { data from } \\
\text { experiment }\end{array}$ & $\begin{array}{c}\text { data from } \\
\text { calculation }\end{array}$ & $\begin{array}{c}\text { data } \\
\text { from } \\
\text { experi } \\
\text { ment }\end{array}$ & $\begin{array}{c}\text { data } \\
\text { from } \\
\text { experim } \\
\text { ent }\end{array}$ & $\begin{array}{c}\text { data from } \\
\text { calculation }\end{array}$ \\
\hline-0.3134 & -0.1360 & 0.4700 & 0.4734 & 1 & 10.0 & 0 & 0.5012 \\
-0.4664 & -0.2030 & 0.7000 & 0.7083 & 1 & 15.0 & 0 & 0.7440 \\
-0.6259 & -0.2730 & 0.9400 & 0.9488 & 1 & 20.0 & 0 & 0.9969 \\
-0.8727 & -0.3800 & 1.3100 & 1.2587 & -4 & 25.0 & 0 & 1.3919 \\
-0.9290 & -0.4200 & 1.4100 & 1.4429 & 2 & 30.0 & 0 & 1.4379 \\
-1.0660 & -0.4640 & 1.6000 & 1.5691 & -2 & 32.0 & 0 & 1.7006 \\
-2.8151 & -2.6000 & 5.6000 & 5.6126 & 0 & 33.6 & 0 & 0.6076 \\
-3.6783 & -3.6000 & 7.5200 & 7.5330 & 0 & 35.6 & 0 & 0.2213 \\
-4.9545 & -5.1200 & 10.4000 & 10.4467 & 0 & 38.4 & 0 & -0.4676 \\
-7.1127 & -7.5200 & 15.1000 & 15.0206 & -1 & 41.6 & 0 & -1.1507 \\
\hline
\end{tabular}

Tab. 1. Axial deformations

As we see, given in the columns 3,4 experimental and calculated sizes of axial deformations practically coincide; their deviations are specified in column 5 . The same results we receive with other materials (aluminium, pig-iron, rocks) [2]. Entering the experimental known data in (10) the reader can be convinced in it. Using the sizes of determined constants, it is possible to calculate other sizes (11), (12) which are given in table 1. In figure 3 loading - deformation and stress - deformation diagrams are resulted. On the loading - deformation diagram experimental $\left(^{*}\right)$ and calculated (o) points are specified. The stress - deformation diagram is constructed according to the (12) formula.

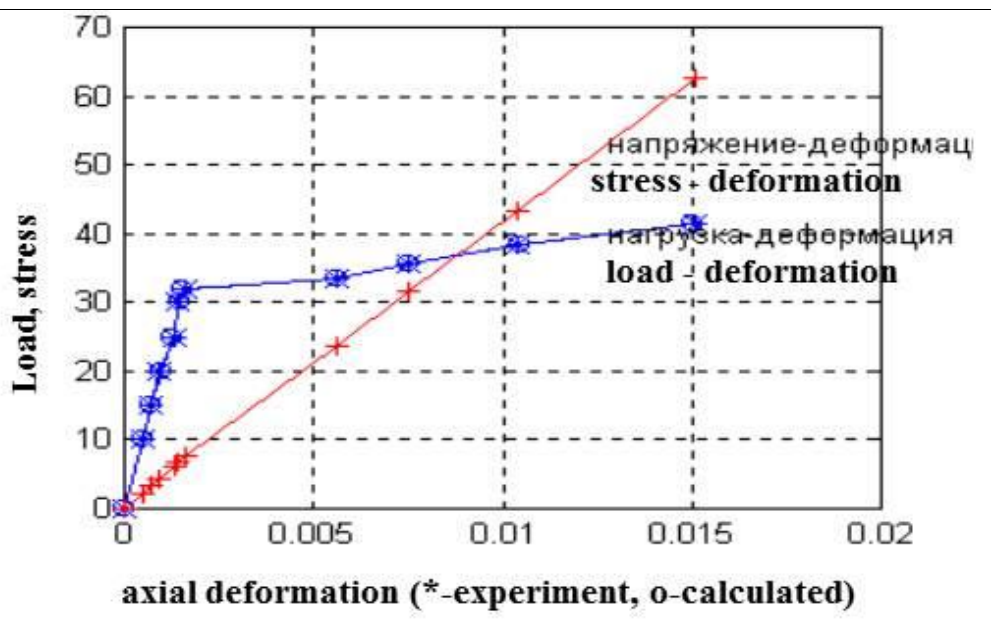

Fig. 3. Load - deformation and stress - deformation diagrams material is steel 30. 
The criterion (5) as the table I and figure 3 overturns our understanding of generalized Hooke's law solvency. It is revealed that this law rather well describes deformation of materials on the whole extent of the diagram at the same constants sizes!

In the rigid body mechanics the diagram loading - deformation is perceived as the diagram stress - deformation or, what practically the same as experimentally determined main law. Meanwhile, as it is seen here, these diagrams differ from each other. This view is epoch-making mistake. It forms a basis of rigid body mechanics for some centuries. The science constructed on such mistake, undoubtedly, is far from true as well as the sky from the earth.

Fig. 4 shows the loading - deformation and stress - deformation of steel 3 diagrams with unloading and loading branches (author's experiments). Experimental and deduced from equation (5) sizes of axis deformation practically coincides, in figure they merge in one point. Constants have the same sizes on the whole diagram.

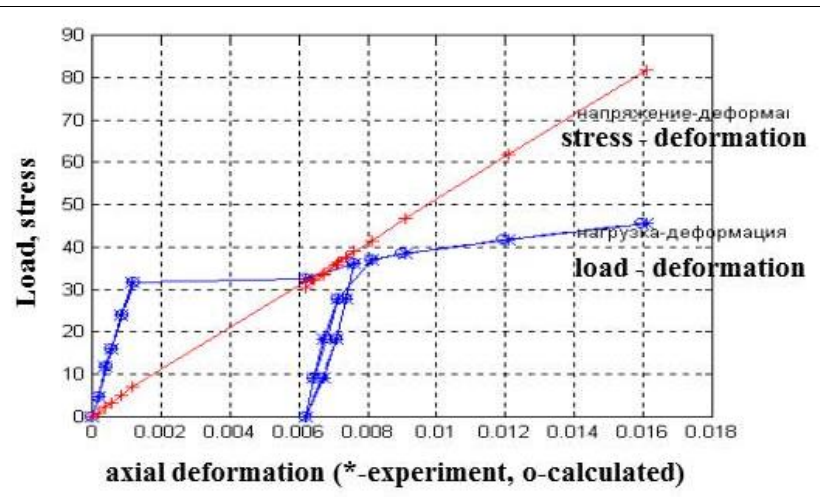

Fig. 4. Load - deformation and stress - deformation diagrams in experiment with unloading (material is steel 3)

Three-axis compression. Radial deformation is defined from a boundary condition on a lateral surface of the cylinder, where liquid stress is applied, which we shall designate through $\operatorname{pr}(\mathrm{t})$. From this condition the deformation is determined as

$$
\varepsilon_{r}(R, 0, t)=\left(p r(t)-\lambda\left(\varepsilon_{\varphi}(R, 0, t)+\varepsilon_{z}(R, 0, t)\right)\right) /(\lambda+2 \mu) .
$$

At axial compression of the cylinder, stress application to its lateral surface, limits development of deformation $\varepsilon_{\varphi}(R, 0, t)$ that complicates the diagram counter. Figure5 shows the loading -deformation diagram of aleurolite [6]. Experimental and deduced from equation (5) points are consequently marked as *, 0 .

In this figure the diagram stress - deformation is also given. Marked with a badge + points correspond to the equation (12). Branches of diagrams are caused by changes of size $\mathbf{g}=\operatorname{pr}(\mathbf{t}) /(\mathbf{P}(\mathbf{t}) /(\pi \mathbf{R 2}))$. In initial four points $\mathrm{g}=0$, further in four points $\mathrm{g}=0.116$, and in last nine points $\mathrm{g}=0.227$.

The equation (5) well describes also the experimental data received in three-axis compression and other rocks (a marble, coal, sandstone, argillite, talk-chlorite, diabase, 
etc.), resulted in work [6]. On the octahedron platform. Generalized Hooke's law at $i \neq j$ get a kind $\sigma_{\mathrm{ij}}=2 \mu \varepsilon_{\mathrm{ij}}$.

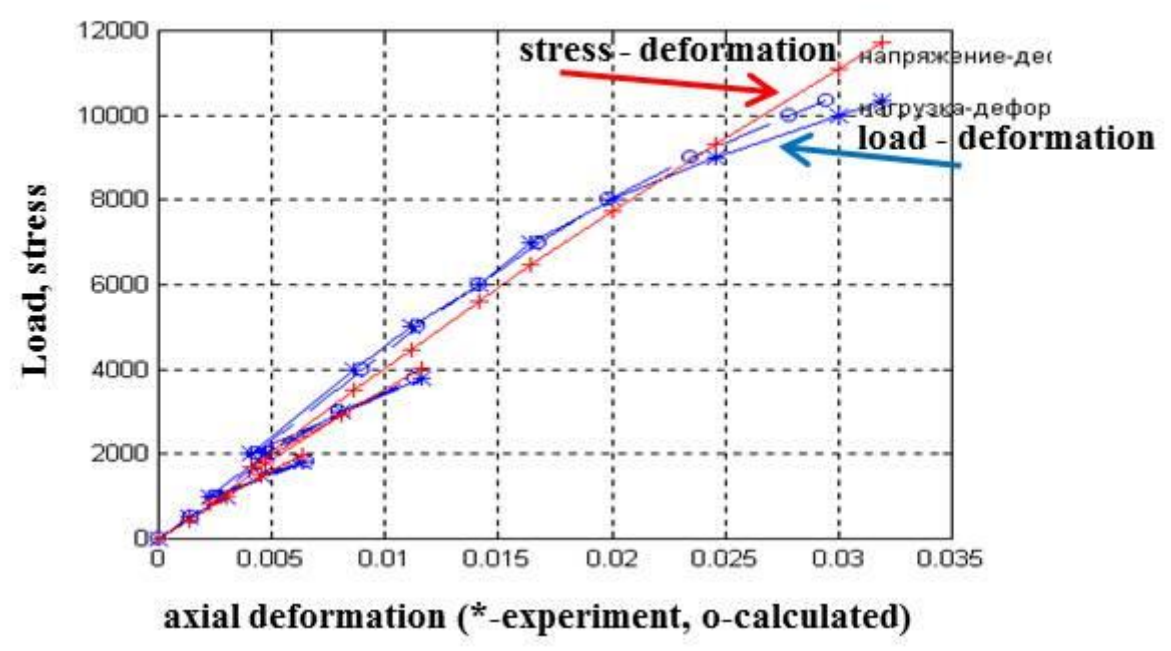

Fig. 5. Comparison of experimental and calculated sizes for aleurolite (three-axis compression)

Tangential stress on any platform is proportionally corresponds to shift. Here diagrams should look like direct lines angular factor of which are to be equal to $2 \mu$. This circumstance we shall consider on octahedron planes. In a point of a cylinder surface $(\mathrm{r}=\mathrm{R}, \mathrm{z}=0)$ all components of a stress and deformation are determined. So, to define the octahedron shift and tangential stress is not difficult. They can be calculated using the formulas:

$$
\begin{aligned}
& \gamma(R, 0, t)=\frac{1}{3} \sqrt{\left(\varepsilon_{r}(R, 0, t)-\varepsilon_{\varphi}(R, 0, t)\right)^{2}+\left(\varepsilon_{\varphi}(R, 0, t)-\varepsilon_{z}(R, 0, t)\right)^{2}+\left(\varepsilon_{z}(R, 0, t)-\varepsilon_{r}(R, 0, t)\right)^{2}} \\
& \tau(R, 0, t)=\frac{1}{3} \sqrt{2\left(\left(\sigma_{\varphi}(R, 0, t)^{2}-\sigma_{\varphi}(R, 0, t) \sigma_{z}(R, 0, t)+\left(\sigma_{z}(R, 0, t)\right)^{2}\right)\right.}
\end{aligned}
$$

Generalized Hooke's law in these designations looks like

$$
\tau(R, 0, t)=2 \mu \gamma(R, 0, t)
$$

On figures 6 and $7 \gamma(R, 0, t), \tau(R, 0, t)$ diagrams for steel 30 and grey pig iron [4] are given. Experimental points here are drawn as a direct line with angular factor $2 \mu$. These lines are bright graphic representation of a solvency of generalized Hooke's law. This surprising solvency disappears, if in expressions (14) we shall make the usual simplifications following from equality (7), (8), and them we shall represent as

$$
\gamma(R, 0)=\frac{\sqrt{2}}{3}\left(\varepsilon_{\varphi}(R, 0)-\varepsilon_{z}(R, 0)\right), \tau(R, 0)=\frac{\sqrt{2}}{3} \frac{P}{\pi R^{2}}
$$


Diagrams $\gamma(R, 0), \tau(R, 0)$ which are given in the same figures, represent widely known curves which form base of many theories $\tau=f(\gamma)$.

However representations (16) are incorrect, they contain the same simplifications, as in usual criterion (8).

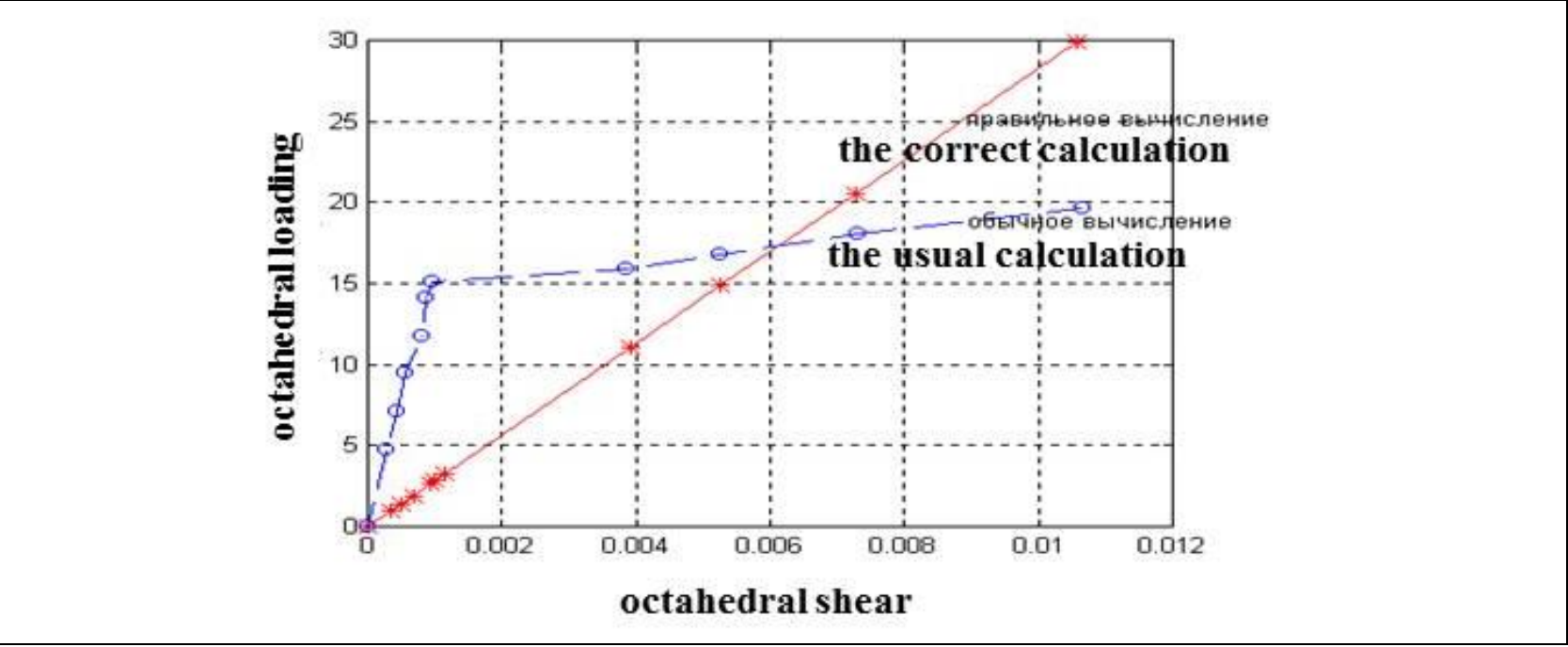

Fig. 6. Dependence between octahedron tangential stress and shear for steel 30 (a straight line $-\gamma(R, 0, t), \tau(R, 0, t)$, a curve - $\gamma(R, 0), \tau(R, 0))$

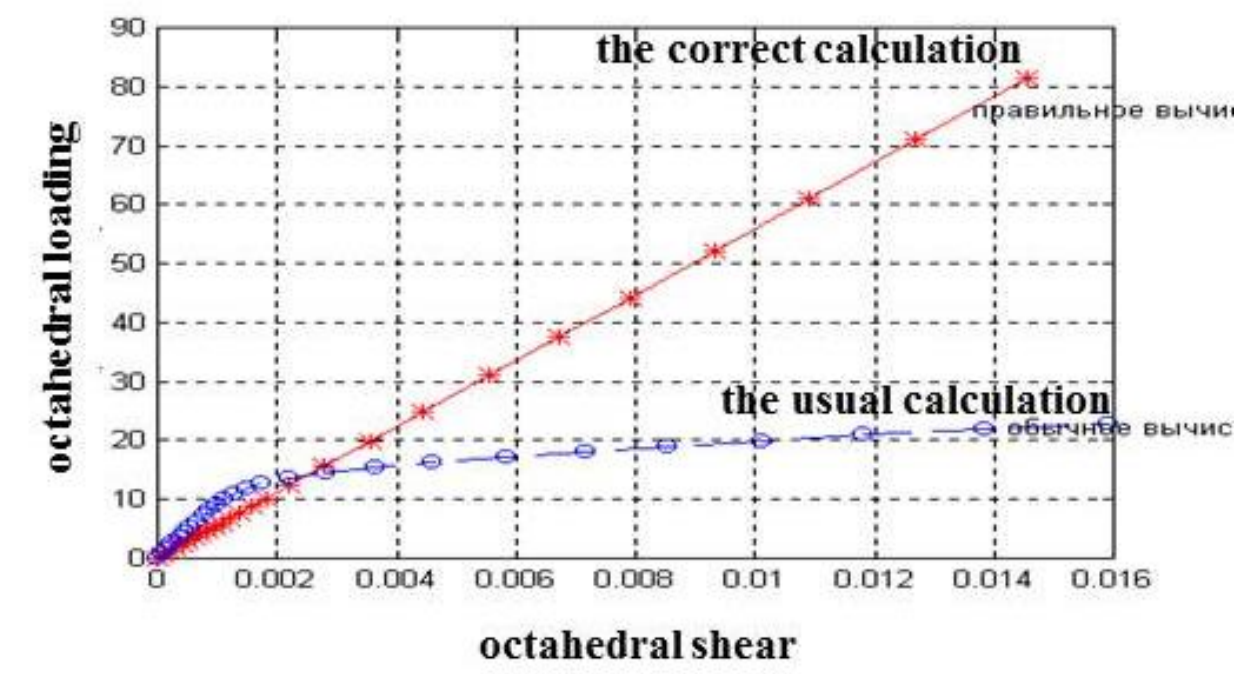

Fig. 7. Dependence between octahedron tangential stress and shift for grey pig-iron (a straight line - $\gamma(R, 0, t), \tau(R, 0, t)$, a curve - $\gamma(R, 0), \tau(R, 0))$.

The Fig. 8 contains the diagram $\gamma(R, 0, t), \tau(R, 0, t)$ for three-axis compression of aleurolite. Here a badge * corresponds to experimental points, a badge $\mathrm{O}$ - to Hooke's law (15).

This diagram represents, perhaps, the brightest illustration of a solvency of the law. This law describes well behavior of a body and at such levels of stress which several times exceed the size of its strength on axial compression! In the three-axis compression second expression (14) looks like 


$$
\tau(R, 0, t)=\frac{1}{3} \sqrt{\left(\sigma_{r}(R, 0, t)-\sigma_{\phi}(R, 0, t)\right)^{2}+\left(\sigma_{\phi}(R, 0, t)-\sigma_{z}(R, 0, t)\right)^{2}+\left(\sigma_{z}(R, 0, t)-\sigma_{r}(R, 0, t)\right)^{2}}
$$

where $\sigma_{r}(R, 0, t)=\operatorname{pr}(t)$ - lateral hydrostatic pressure.

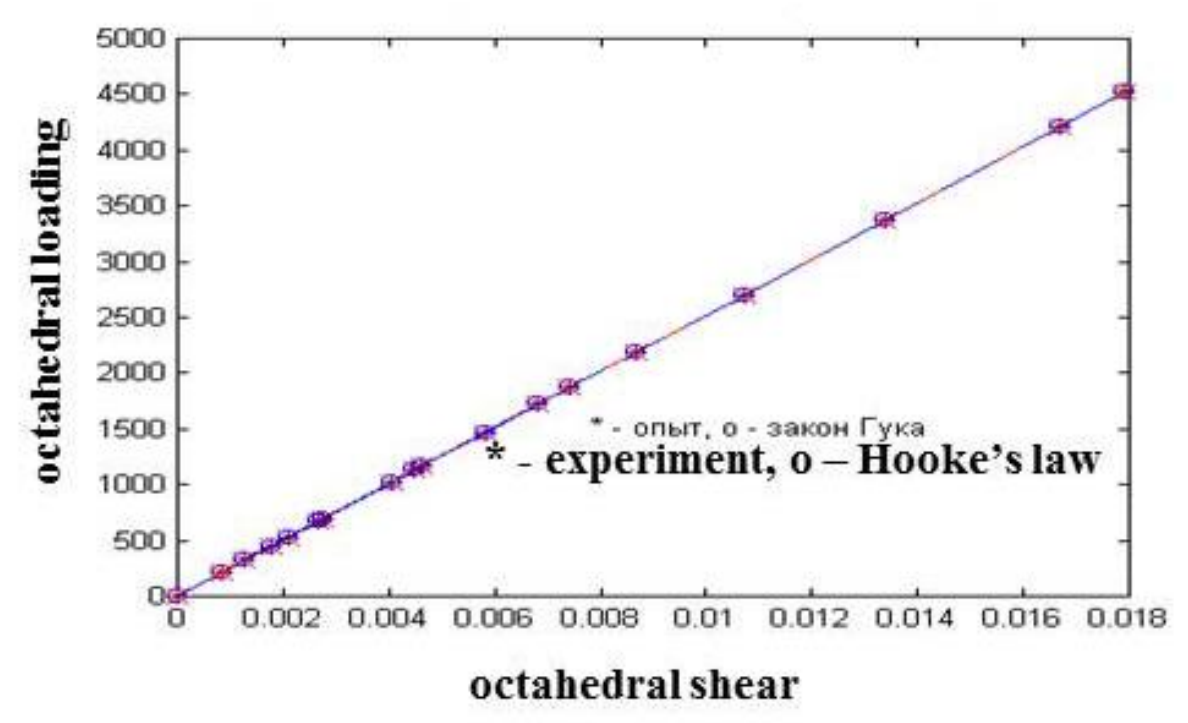

Fig. 8. Arrangements of experimental points on octahedron planes for three axis compression of aleurolite.

\section{About distribution of an axial stress in cross-section}

In the points located on a contour of the central cross-section, the deformations $\varepsilon_{\varphi}(R, 0, t), \varepsilon_{z}(R, 0, t)$ are measured. A third to a component of deformation $\varepsilon_{r}(R, 0, t)$ one may define using the expression (11) received from a boundary condition $\sigma_{r}(R, 0, t)=0$. Further, using formulas (12), one may calculate $\sigma_{\varphi}(R, 0, t), \sigma_{z}(R, 0, t)$. These data are resulted in table 1 . Here we see that

$\sigma_{\varphi}(R, 0, t) \neq 0, \sigma_{z}(R, 0, t) \neq \frac{P(t)}{\pi R^{2}}$

it rejects equality (7). Real intensive test conditions for axial compression or a stretching are not one-axis.

The inequality (table I)

$$
\varepsilon_{r}(R, 0, t) \neq \varepsilon_{\varphi}(R, 0, t)
$$

is the obvious sign of non-one axis. This inequality allows distributing of an axial stress to cross-section. We shall consider, for example, axial compression. In an initial part 
Duishenaliev, T.; Duishenaliev, C. \& Mekenbaev, B.: The Equation of Load Defor...

of the diagram

$\varepsilon_{r}(R, 0, t)>\varepsilon_{\varphi}(R, 0, t)$

It is natural to assume, that the inequality takes place in internal points also

$$
\frac{\partial u(r, 0, t)}{\partial r}>\frac{u(r, 0, t)}{r}
$$

Further we shall believe, function $u(r, 0, t)$ and deformations $\frac{\partial u(r, 0, t)}{\partial r}, \frac{u(r, 0, t)}{r}$ are continuous in an interval $0 \leq r \leq R$. Multiplying both parts of this inequality on $\mathrm{r}$ and, differentiating on $\mathrm{r}$, we come

$\frac{\partial^{2} u(r, 0, t)}{\partial r^{2}}>0$

This inequality shows that in this stage of deformation, the function $u(r, 0, t)$ a concaved up curve (figure 9).

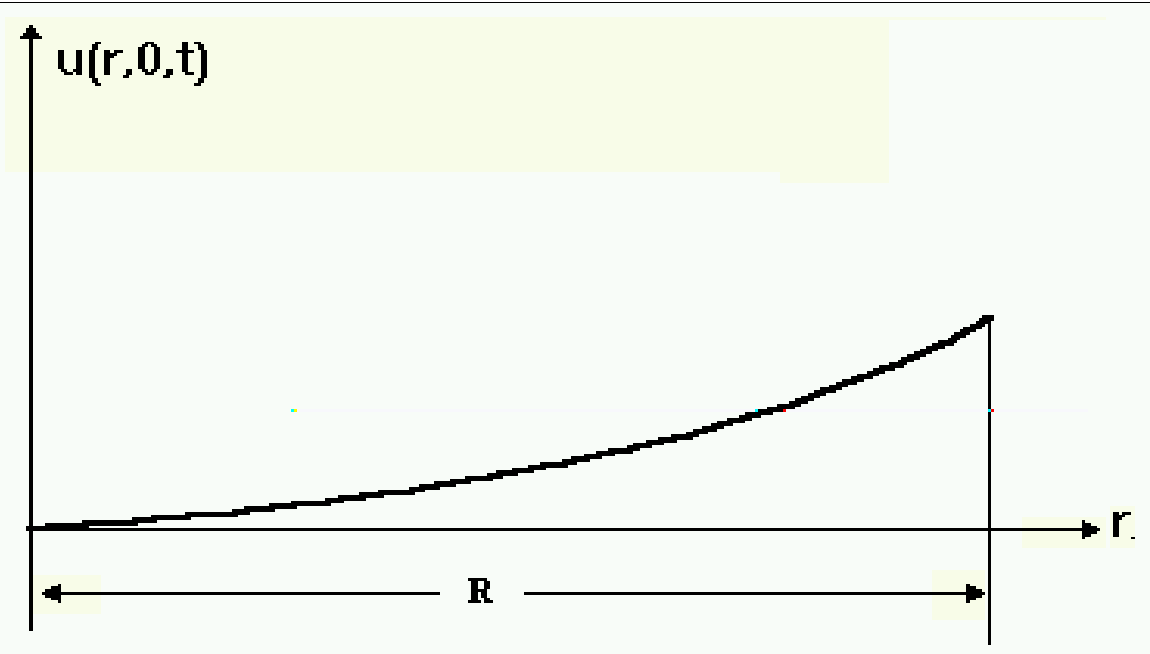

Fig. 9. Character of function $u(r, 0, t)$ in an initial part of the diagram.

For such curve $u(r, 0, t)$ each addendum of the sums

$\frac{\partial u(r, 0, t)}{\partial r}+\frac{u(r, 0, t)}{r}$

grows with $r$ increasing. In that case, an axial stress

$$
\sigma_{z}(r, 0, t)=\lambda(+) \frac{\partial u(r, 0, t)}{\partial r} \frac{u(r, 0, t)}{r}+(\lambda+2 \mu) \frac{\partial w(R, 0, t)}{\partial z}
$$


decreases with $\mathrm{r}$ increasing (signs $\frac{\partial u(r, 0, t)}{\partial r}+\frac{u(r, 0, t)}{r}$ and $\frac{\partial w(R, 0, t)}{\partial z}$ are opposite). Thus distribution $\sigma_{z}(r, 0, t)$ will look like, shown on figure 10 a. Here we shall repeat a reason which was used for representation $\varepsilon_{z}(r, 0, t)=\varepsilon_{z}(R, 0, t)=\frac{\partial w(R, 0, t)}{\partial z}$.

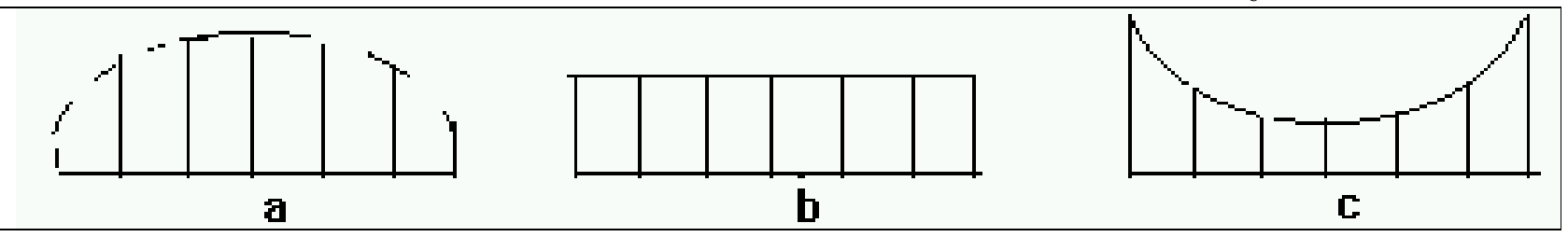

Fig. 10. Character of distribution $\sigma_{z}(r, 0, t)$ in an initial part of the diagram (a), in a one axis point (b) and in a final part of the diagram (c).

Function $w(r, z, t)$ because of symmetry of loadings at $z=0$ does not depend from $\mathrm{r}$ $w(r, 0, t)=0$.

The central cross-section section remains flat. Here it is possible to believe, as at values $\mathrm{z}$, close to zero, function $w(r, z, t)$ depends from $\mathrm{r}$ very little, and the mentioned above representation allows it. In a point of one axis

$\varepsilon_{r}(R, 0, t)=\varepsilon_{\varphi}(R, 0, t)$.

If such equality takes place in internal points, then

$\frac{\partial u(r, 0, t)}{\partial r}=\frac{u(r, 0, t)}{r}$

Thus movement function $u(r, 0, t)$ represents a direct line, for which $\frac{\partial u(r, 0, t)}{\partial r}+\frac{u(r, 0, t)}{r}=$ const. In this case, the right part (18) does not depend from $\mathbf{r}$ i.e. $\sigma_{z}(r, 0, t)=$ const (figure $10 \mathrm{~b}$ ). In a final part of the diagram $\varepsilon_{r}(R, 0, t)>\varepsilon_{\varphi}(R, 0, t)$. If inside the section we have the same picture, then $\frac{\partial u(r, 0, t)}{\partial r}<\frac{u(r, 0, t)}{r}$. Acting the similar way, we find $\frac{\partial^{2} u(r, 0, t)}{\partial r^{2}}<0$

This inequality shows that in this stage of deformation, the function $u(r, 0, t)$ concave down curve (figure 11). For such curve $u(r, 0, t)$ each addendum of the sum $\frac{\partial u(r, 0, t)}{\partial r}+\frac{u(r, 0, t)}{r}$

decreases with $\mathrm{r}$ increasing. In that case, an axial stress $\sigma_{z}(r, 0, t)$, apparently from (37), increases with $\mathrm{r}$ increasing (figure 10, c). 
Such character of distribution $\sigma_{z}(r, 0, t)$ proves the data given in table 1 . We see, that $\sigma_{z}(R, 0, t)<\frac{P(t)}{\pi R^{2}}$ in an initial part of the diagram, in a point of one - axis $\sigma_{z}(R, 0, t)=\frac{P(t)}{\pi R^{2}}$ , and in a final part $\sigma_{z}(R, 0, t)>\frac{P(t)}{\pi R^{2}}$.

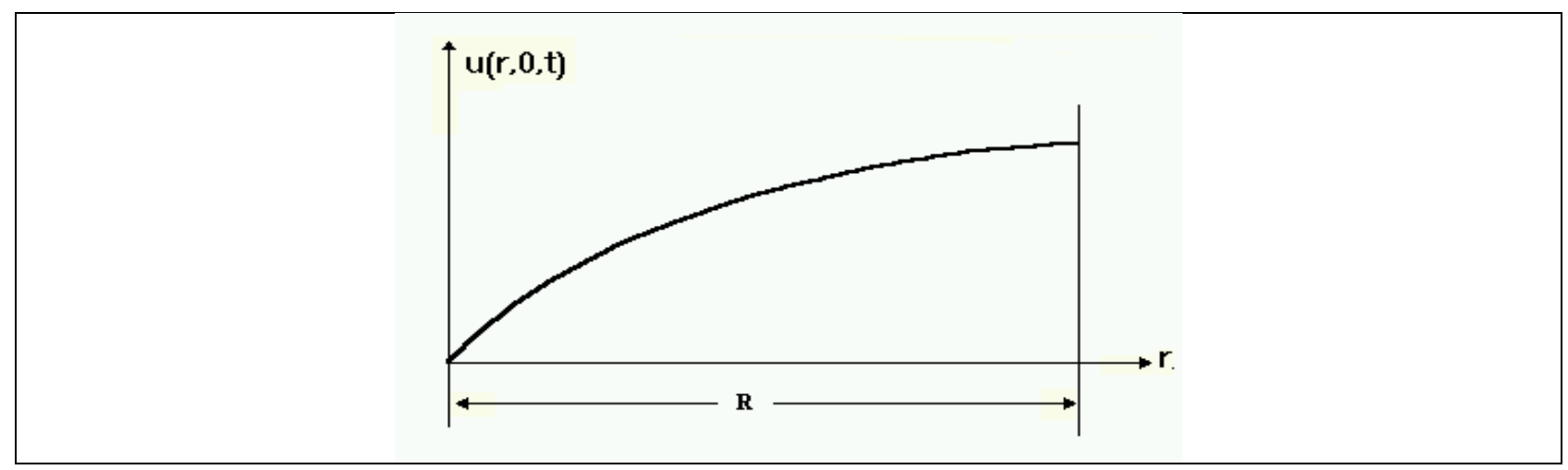

Fig. 11. Character of function $u(r, 0, t)$ in a final part of the diagram

\section{About constants sizes determined on the basis of usual and new criterions}

The axial stress on cross-section section, as shown above, is distributed non-uniformly. In the loading process this distribution undergoes the evolution shown on figure 10. This circumstance rejects equality (7) on which during the all loading time, the axial stress on cross-section section is distributed in regular intervals with intensity of $P / \pi R^{2}$.

As shown above, at the moment, when $\varepsilon_{r}(R, 0, t)=\varepsilon_{\varphi}(R, 0, t)$, uniform distribution of an axial stress to cross-section section is possible.

Thus equalities (7) and, the following them, usual criterion (8), get legitimacy. In all other points of the diagram $\varepsilon_{r}(R, 0, t) \neq \varepsilon_{\varphi}(R, 0, t)$, that makes an intense condition on the perimeter of cross-section section not one-axis. In this case, the equalities (7), and the ratio (8), lose a force.

Thus, the usual criterion truly defines constants $\mathbf{E}, \boldsymbol{v}$, only in that point of the diagram, where $\varepsilon_{r}(R, 0, t)=\varepsilon_{\varphi}(R, 0, t)$. In other points it gives false results.

It is not an ordinary conclusion, as it is followed, that the sizes of these constants, registered in the educational and scientific literature and for some centuries used in technical and design calculations, are defined incorrectly. Now we are talking about unimaginably big mistakes. So, elastic modulus, determined by new criterion, has much smaller sizes! And Poisson ratio, on the contrary, has unusually large sizes.

The data of table I prove the correctness of new sizes $\mathbf{E}, \boldsymbol{v}$. They remain the same at replacement $\lambda, \mu$ on $\mathbf{E}, \boldsymbol{v}$ in all used there. Thus, for example, the equation (5) will look like 


$$
\varepsilon_{z}(R, 0, t)=\frac{(1+v)(1-2 v)}{E(1-v)}\left(\frac{P(t)}{\pi R^{2}}-\frac{2 E v}{(1+v)(1-2 v)} \varepsilon_{\varphi}(R, 0, t)\right)
$$

Let's define constants $\mathbf{E}, \boldsymbol{v}$, using usual criterion (8). Shall we come to the values that are established by new criterion? If yes, it should be accepted as one more proof of new values of these constants.

So, the usual criterion is applicable only at the moment of time $\mathbf{t}=\mathbf{t}_{\mathbf{0}}$ when the intense condition in a point $\mathbf{r}=\mathbf{R}, \mathbf{z}=\mathbf{0}$ becomes one-axis. Thus

$$
\sigma_{r}\left(R, 0, t_{0}\right)=\sigma_{\varphi}\left(R, 0, t_{0}\right)=0, \quad \sigma_{z}\left(R, 0, t_{0}\right)=\frac{P\left(t_{0}\right)}{\pi R^{2}}
$$

From here ratio follow

$$
\varepsilon_{r}\left(R, 0, t_{0}\right)=\varepsilon_{\varphi}\left(R, 0, t_{0}\right)=-\frac{v}{E} \frac{P\left(t_{0}\right)}{\pi R^{2}}, \quad \varepsilon_{z}\left(R, 0, t_{0}\right)=\frac{1}{E} \frac{P\left(t_{0}\right)}{\pi R^{2}}
$$

At this moment an axial stress

$$
\sigma_{z}\left(R, 0, t_{0}\right)=\lambda\left(\varepsilon_{r}\left(R, 0, t_{0}\right)+\varepsilon_{\varphi}\left(R, 0, t_{0}\right)\right)+(\lambda+2 \mu) \varepsilon_{z}\left(R, 0, t_{0}\right)
$$

because of $\varepsilon_{r}\left(R, 0, t_{0}\right)=\varepsilon_{\varphi}\left(R, 0, t_{0}\right)$, can be shown as

$$
\sigma_{z}\left(R, 0, t_{0}\right)=2 \lambda \varepsilon_{\varphi}\left(R, 0, t_{0}\right)+(\lambda+2 \mu) \varepsilon_{z}\left(R, 0, t_{0}\right)
$$

From here, taking into account (19), we have

$$
\varepsilon_{\varphi}\left(R, 0, t_{0}\right)=\frac{1}{2 \lambda}\left(\frac{P\left(t_{0}\right)}{\pi R^{2}}-(\lambda+2 \mu) \varepsilon_{z}\left(R, 0, t_{0}\right)\right)
$$

Elastic Modulus and Poisson ratio now can be defined under the following formulas

$$
E=\frac{P\left(t_{0}\right)}{\pi R^{2} \varepsilon_{z}\left(R, 0, t_{0}\right)}, v=-\frac{\frac{P\left(t_{0}\right)}{\pi R^{2}}-(\lambda+2 \mu) \varepsilon_{z}\left(R, 0, t_{0}\right)}{2 \lambda \varepsilon_{z}\left(R, 0, t_{0}\right)}
$$

Further, it is necessary to find sizes $P\left(t_{0}\right) / \pi R^{2}, \varepsilon_{z}\left(R, 0, t_{0}\right)$. At the moment to diagrams on figure 3 are crossed, as they, in this case, have the same ordinate equal to $P\left(t_{0}\right) / \pi R^{2}$ 
. We use the operator ginput for this point as

$$
\left[\varepsilon_{z}\left(R, 0, t_{0}\right), \frac{P\left(t_{0}\right)}{\pi R^{2}}\right]=\text { ginput }
$$

also we determine

$$
\varepsilon_{z}\left(R, 0, t_{0}\right)=0.0088, \frac{P\left(t_{0}\right)}{\pi R^{2}}=36.84
$$

Substituting these sizes in (21), (22), we find

$$
\varepsilon_{\varphi}\left(R, 0, t_{0}\right)=-0.0043, \quad E=4192, \quad v=0.4841
$$

These values of elastic modulus and Poisson ratio are rather close to those their sizes that are determined from a position of criterion (5). To the same results we come and in the case of other materials (see table II).

\begin{tabular}{|l|c|c|c|c|c|l|l|l|}
\hline \multicolumn{1}{|c|}{ Name } & \multicolumn{2}{|c|}{$\begin{array}{c}\text { Definitions on } \\
\text { coordinates of a } \\
\text { one-axis point }\end{array}$} & \multicolumn{2}{|c|}{$\begin{array}{c}\text { Definitions by } \\
\text { new criterion }\end{array}$} & \multicolumn{4}{|c|}{ Coordinates of one-axis point } \\
\hline & $\mathbf{E}$ & $\mathbf{v}$ & $\mathbf{E}$ & $\mathbf{v}$ & $\varepsilon_{\varphi}\left(R, 0, t_{0}\right)$ & $\varepsilon_{z}\left(R, 0, t_{0}\right)$ & $\varepsilon_{z}\left(R, 0, t_{0}\right)$ & $\varepsilon_{z}\left(R, 0, t_{0}\right)$ \\
\hline Steel 30 & 4192 & 0.4841 & 4193 & 0.4841 & -0.0043 & 0.0088 & 0.0088 & 0.0088 \\
\hline Aluminum & 1620 & 0.4796 & 1613 & 0.4799 & -0.0028 & 0.0058 & 0.0058 & 0.0058 \\
\hline $\begin{array}{l}\text { Grey pig- } \\
\text { iron }\end{array}$ & 7989 & 0.4144 & 7922 & 0.4168 & 0.0016 & -0.0038 & -0.0038 & -0.0038 \\
\hline
\end{tabular}

Tab. 2. Coordinates of one-axis point

So, from table II it is visible, that in a one-axis point ratio (8) truly define sizes $\mathbf{E}, \boldsymbol{v}$. In other points they lose force. Definitions of constants, using these data, should lead to the large mistakes. The longer the distance between points and one-axis, the larger the mistake. Such distance increases a degree of non-uniformity of distribution of an axial stress on cross-section section. In the rigid body mechanics, a ratio (16) is traditionally used in an initial part of the diagram, where an inequality $\varepsilon_{r}(R, 0, t) \neq \varepsilon_{\varphi}(R, 0, t)$ has the most strongly expressed character. If to judge from the equation (5), determined sizes of so-called tangential or secant module, most likely, are closer to size $\lambda+2 \mu$, rather than $\mathbf{E}$.

\section{About mistakes in mechanics of a deformed body, caused by simplifications}

The usual criterion (8) is direct consequence of boundary conditions (6). If these conditions are erroneous, the usual criterion loses force. First of conditions (8) is 
allowable, it is based on Saint-Venant narrowing principle which is proved theoretically. Second of boundary conditions (6) is based on common Saint-Venant principle which has no the theoretical proof. Numerous attempts, as is known, had no success. From here follows, that this condition nevertheless is doubtful. The inconsistency of this condition shows also new criterion. Distribution of size of an axial stress to cross-section section is far from uniformity. Besides, as shown in point 5, character of distribution changes depending on size of axial loading. In view of it, it is necessary to recognize second of conditions (6) insolvent. If it so, equality (7) are not the decision of a static regional task. The criterion (8) disappears, for it is received from these equalities. It is important, but a unpleasant conclusion. Thus, the solvency of generalized Hooke's law, since time of its occurrence, is found out incorrectly. Both the conclusions and definitions are incorrect also. These definitions and conclusions are the basis on which rigid body mechanics has been constructed.

Definition of constants as

$$
E=P /\left(\varepsilon_{z} \pi R^{2}\right), v=-\varepsilon_{\varphi} / \varepsilon_{z},
$$

interpretation of loading - deformation diagrams as certain laws,

$$
\sigma_{z}=f\left(\varepsilon_{z}\right), \tau=g(\gamma),
$$

have fluid mechanics of a deformed body with various views, hypotheses, postulates, concepts, models and theories. From the definitions (24), it becomes clear, that the body continually changes sizes of its constants. It is equivalent to, figuratively speaking, that silver turns to tin or that a giant, changing an Armour, becomes the dwarf and vice versa. The experimental diagrams perceived as laws (25), are divided into peculiar parts, which have their names. Even their separate bends and inclinations have names There is no the uniform mathematical description of the diagram, in spite of the fact that brilliant mathematicians and mechanics (Lagrange, Cauchy, Poisson, Ljav, Maxwell, Einstein, Born) tried to elaborate it. The diagram with branches of unloading has entered (25) terrible diversity into the functions. The extremely complicated application of functions (25) in three-dimensional tasks has appeared. This is the exact condition of a rigid body mechanics, which is created by using of usual criterion.

However, everything is not so complicated. The experimental loading deformation diagram is described by the equation (5). This equation is surprisingly simple equation, but, nevertheless, it traces all parts of the diagram, including its branches of unloading, at the same constants sizes. Interpretation of this diagram as determined by the experience law, between a stress and deformation as it is found out here, is wrong. Hence, everything that was elaborated on this basis is also wrong, that is, practically the whole essence of the rigid body mechanics, for this science was developed in an atmosphere of such interpretation. 


\section{Conclusion}

From the point of view of usual criterion the body submits to generalize Hooke's law if its experimental diagram from the beginning up to the end is rectilinear. But the diagram is not rectilinear. It is curvilinear, has rather complex contours. Hence, this law, in general, is not solvent. It is possible to apply its initial part of the diagram, if this part is rectilinear or close to this. Here it is necessary to note, that straightforwardness of an initial part is a disputable position. The diagram here again has curvature. As academician J.N. Rabotnov noticed, it can be found out even at small deformations. The more perfect the measuring equipment, the earlier it is possible. It has led him to the conclusion that in the field of small deformations there is a regular deviation from the law [5]. From its solvency, thus, remained nothing. It is the reputation of generalized Hooke's law, which is created by usual criterion.

The new criterion completely destroys this state of affairs! It will rehabilitate this law, erects it in a rank of fundamental laws of the nature. Rather precisely describing behaviour of a body on all an extent of the diagram, it quite justifies both this status, and the strict physical substantiation.

\section{References}

M. Born, Huan Kun. The dynamic theory of crystal lattices. M., IL, 1958, p. 488.

A.B. Jakypbek, T.B. Duyishenaliev .New view on some bases of mechanics of a deformed body. Bishkek, 1999.

A.M. Jukov. About Poisson ratio in the plastic area. News of the AS USSR, OTN, 1954, № 12.

B.A. Panjaev. About the destruction mechanics of semi-brittle bodies. The dissertation for candidate of sciences. Frunze, 1974.

Y.N. Rabotnov. Mechanics of materials M., Physmatgiz, 1962, page 128.

A.N. Stavrogin, A.G. Protesenja. Plasticity of rocks. M., Nedra, 1981. 